\title{
BUILDING A LUNAR OR MARTIAN LAUNCH PAD WITH IN SITU MATERIALS: RECENT LABORATORY AND FIELD STUDIES
}

Paul E. Hintze ${ }^{1}$ and Stephanie Quintana ${ }^{2}$

${ }^{1}$ Chemical Analysis Branch, NASA Kennedy Space Center, FL 32899

2 Planetary Geoscience Group, Brown University, Providence, RI 02912

\section{Overview}

- Why do we need launch and landing pads?

- Methods

- Effects of using different simulants

- Recent field studies_.

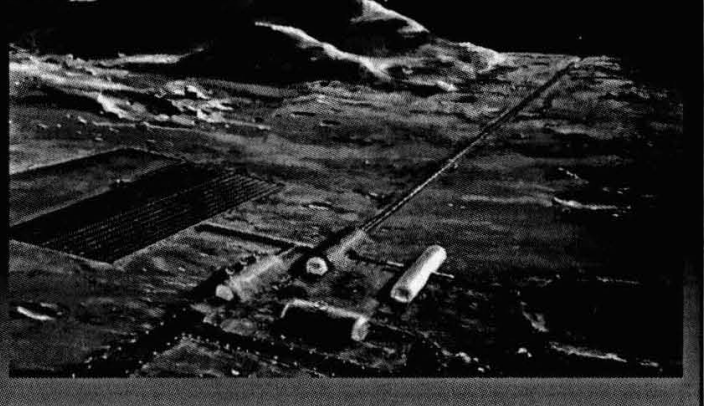




\section{Dust and surface stabilization}

- Dust ejecta during lunar launch/landing can affect visibility, erode nearby coated surfaces and get into mechanical assemblies of in-place infrastructure

- Dust mitigation will necessary for certain lunar habitat

- Surface stabilization can be used for roads, pads and other free areas

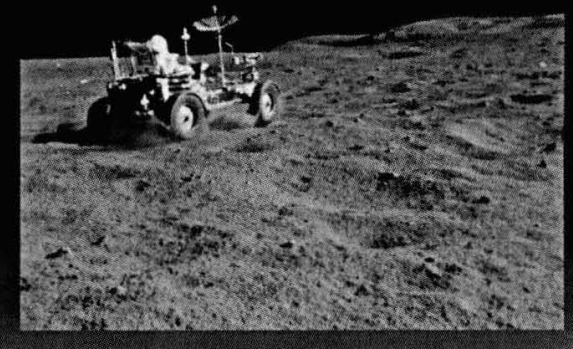

\section{Surveyor III}

- Apollo 12 LM landed 155m from the Surveyor III craft

- NASA-SP-284: Analysis of Surveyor 3 material and photographs returned by Apollo 12 - found "sandblasting" with shadows showing that the blast came from the LM

- Immer et al. Icarus 2010, 2011; Lane et al. this conference

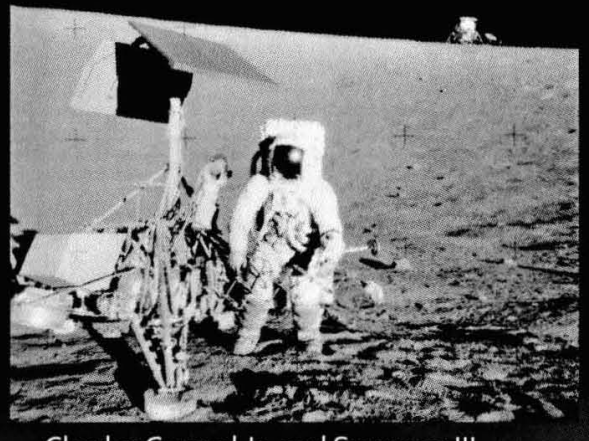

Charles Conrad Jr. and Surveyor III 


\section{Stabilization Methods}

- Polymer regolith Composites

- Sintering/melting regolith

- Others...

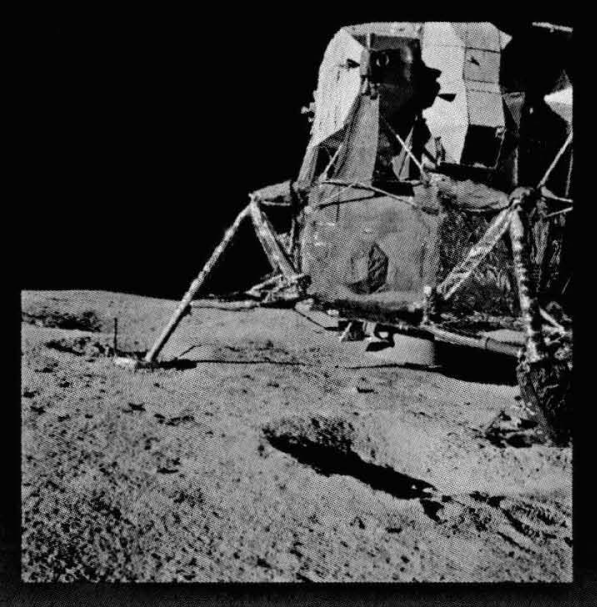

Simulant Laboratory Study: Sintering

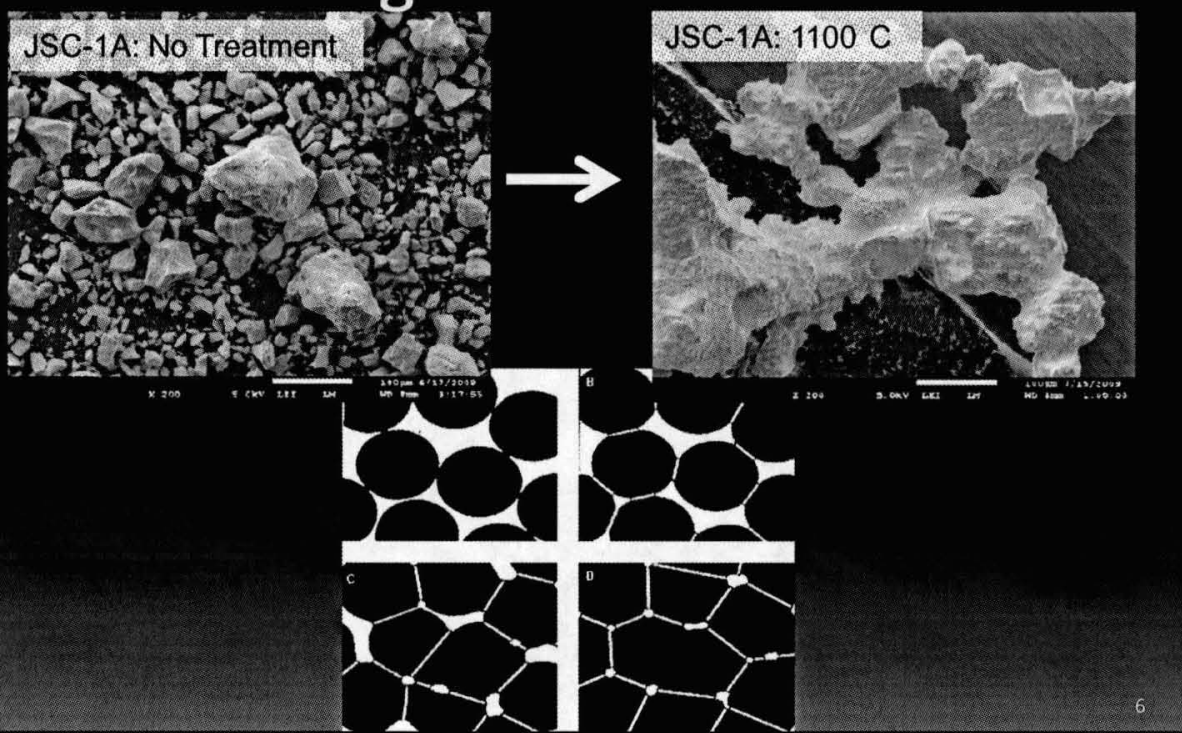




\section{Simulant Laboratory Study:}

\section{Simulants}

- FJS-1, Japanese Mare

- JSC-1A, Orbitec Mare

- NU-LHT-2M, NASA/USGS Highlands

- OB1, NORCAT

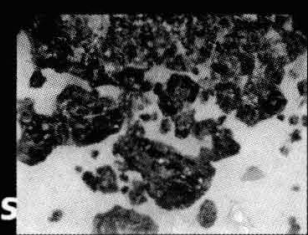

FJS-1 Highlands

- Heated samples to $1000,1050,1100,1150$ and $1200 \mathrm{C}$ in nitrogen purged furnace

- Use elemental analysis to identify individual grains and check for changes after heating

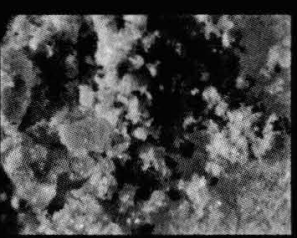

NU-LHT-2M

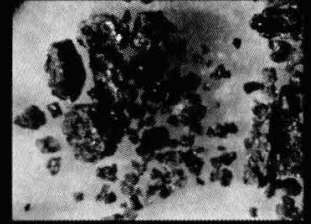

JSC- $1 \mathrm{~A}$

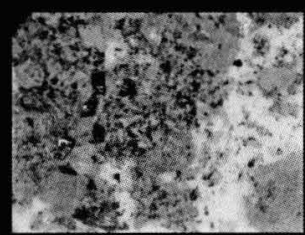

OB-1

\section{Simulant Laboratory Study:} Analysis

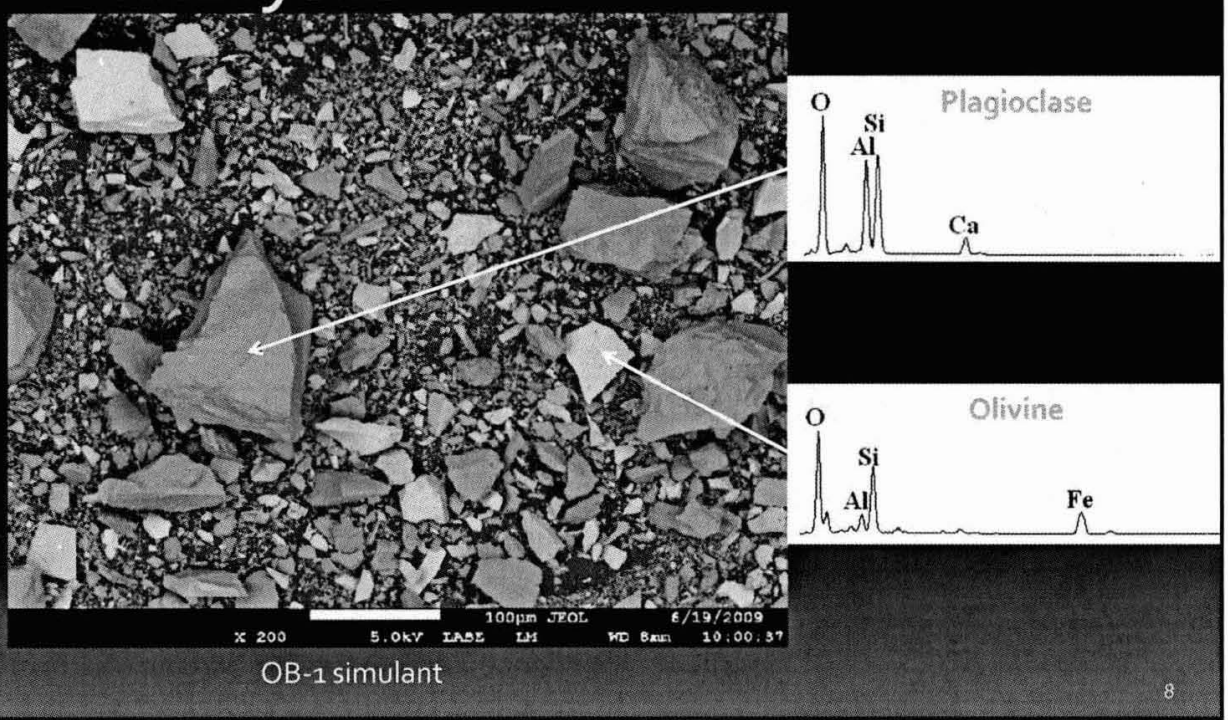




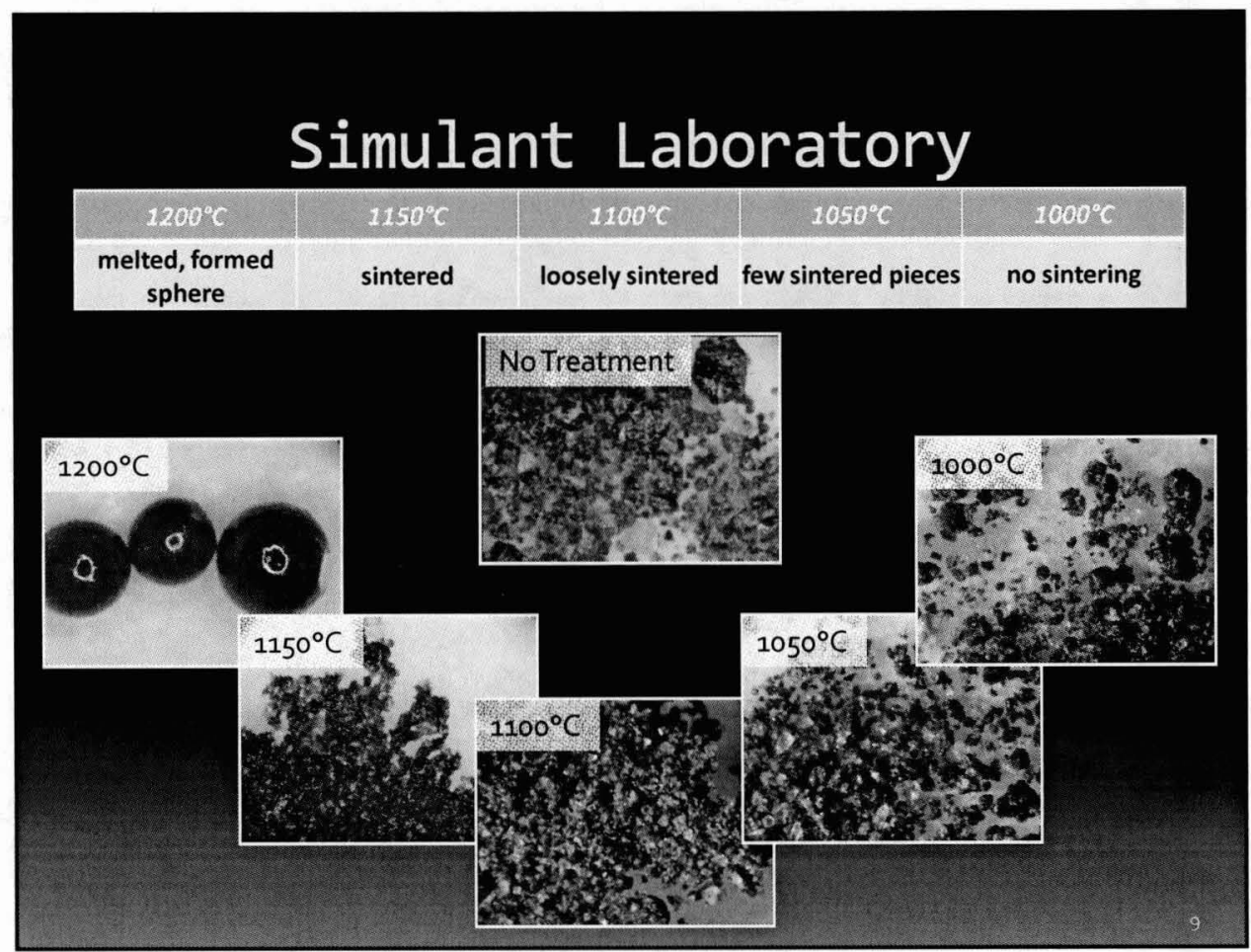

Simulant Laboratory Study: FJS-1

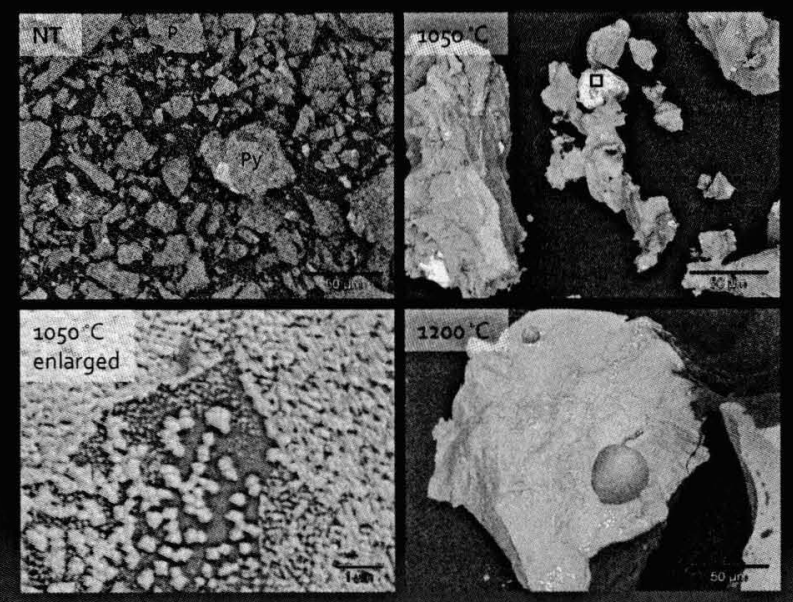




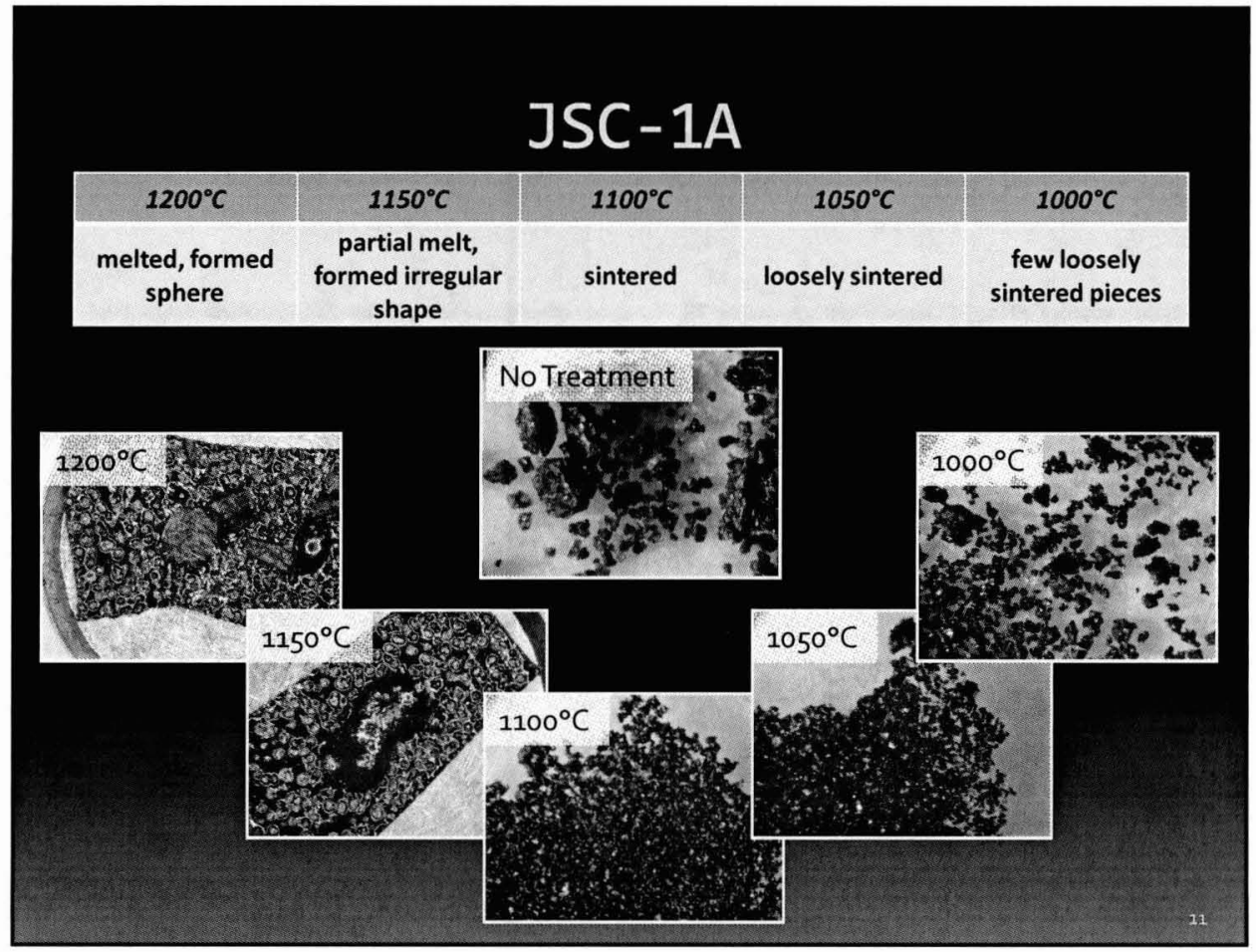

\section{Simulant Laboratory Study: JSC - $1 \mathrm{~A}$}

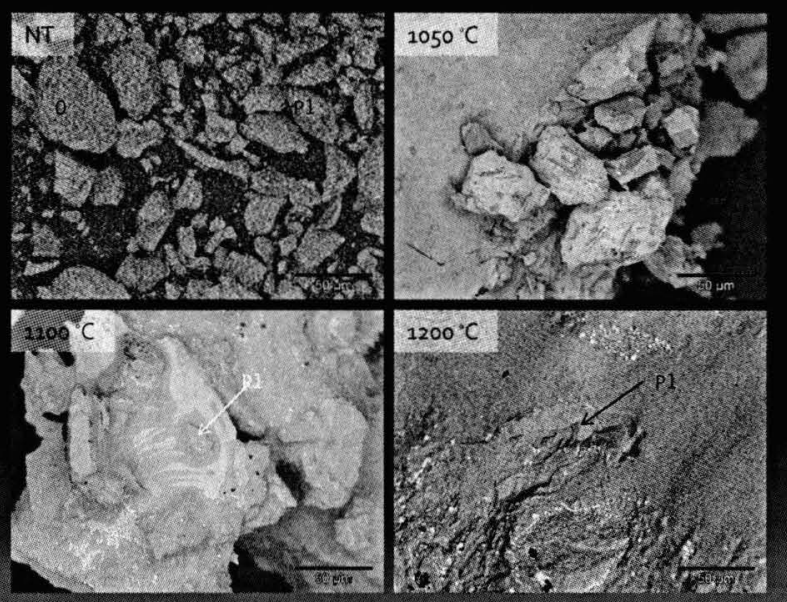




\section{NU-LHT-2M}

\begin{tabular}{|c|c|c|c|c|}
\hline $1200^{\circ} \mathrm{C}$ & $1150^{\circ} \mathrm{C}$ & $1100^{\circ} \mathrm{C}$ & $1050^{\circ} \mathrm{C}$ & $1000^{\circ} \mathrm{C}$ \\
\hline sintered & sintered & few sintered pieces & no sintering & no treatment \\
\hline
\end{tabular}

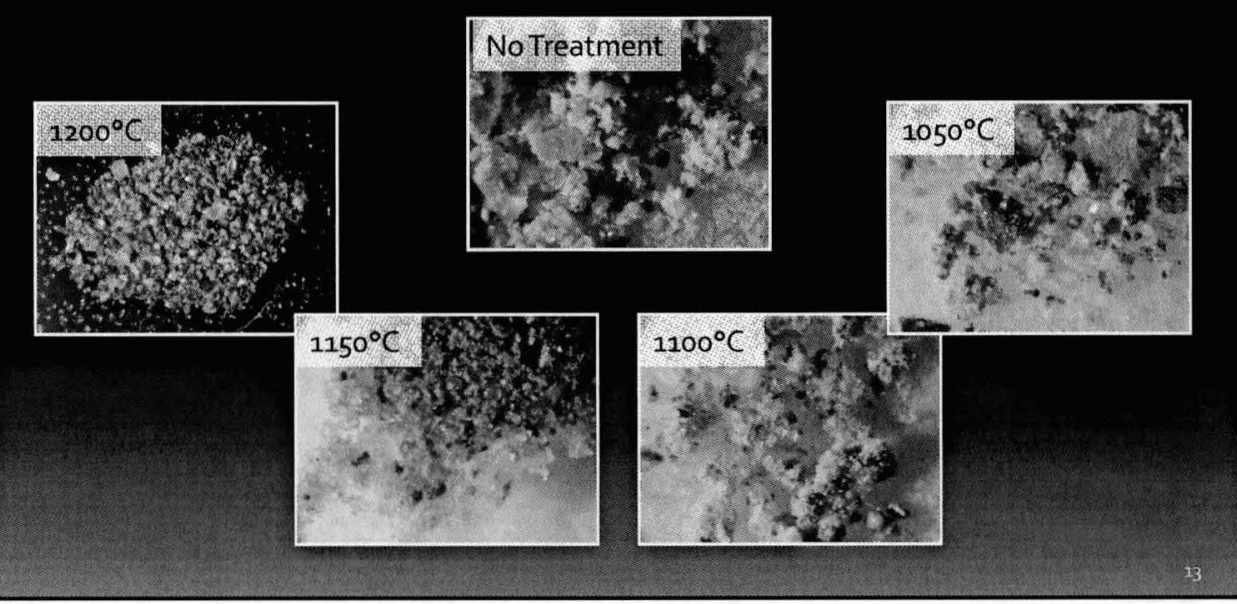

Simulant Laboratory Study: NU-LHT-2M

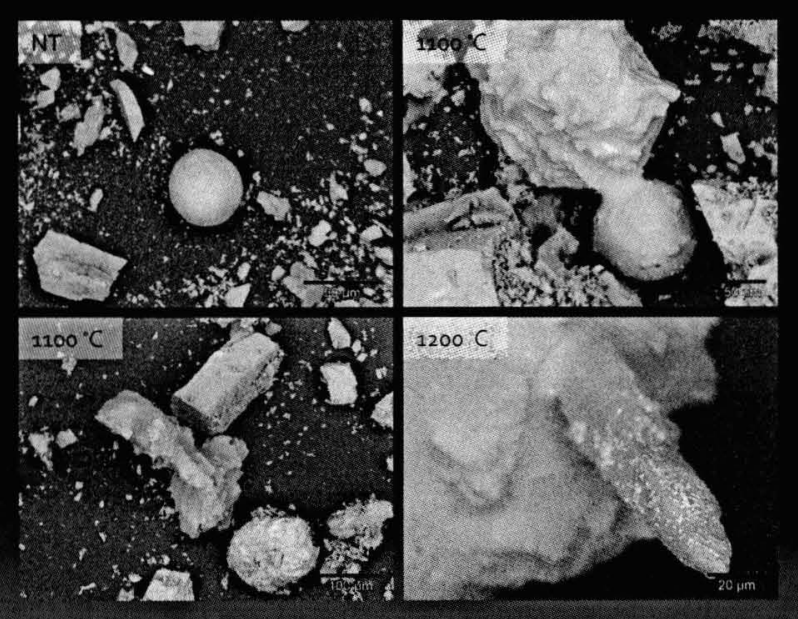




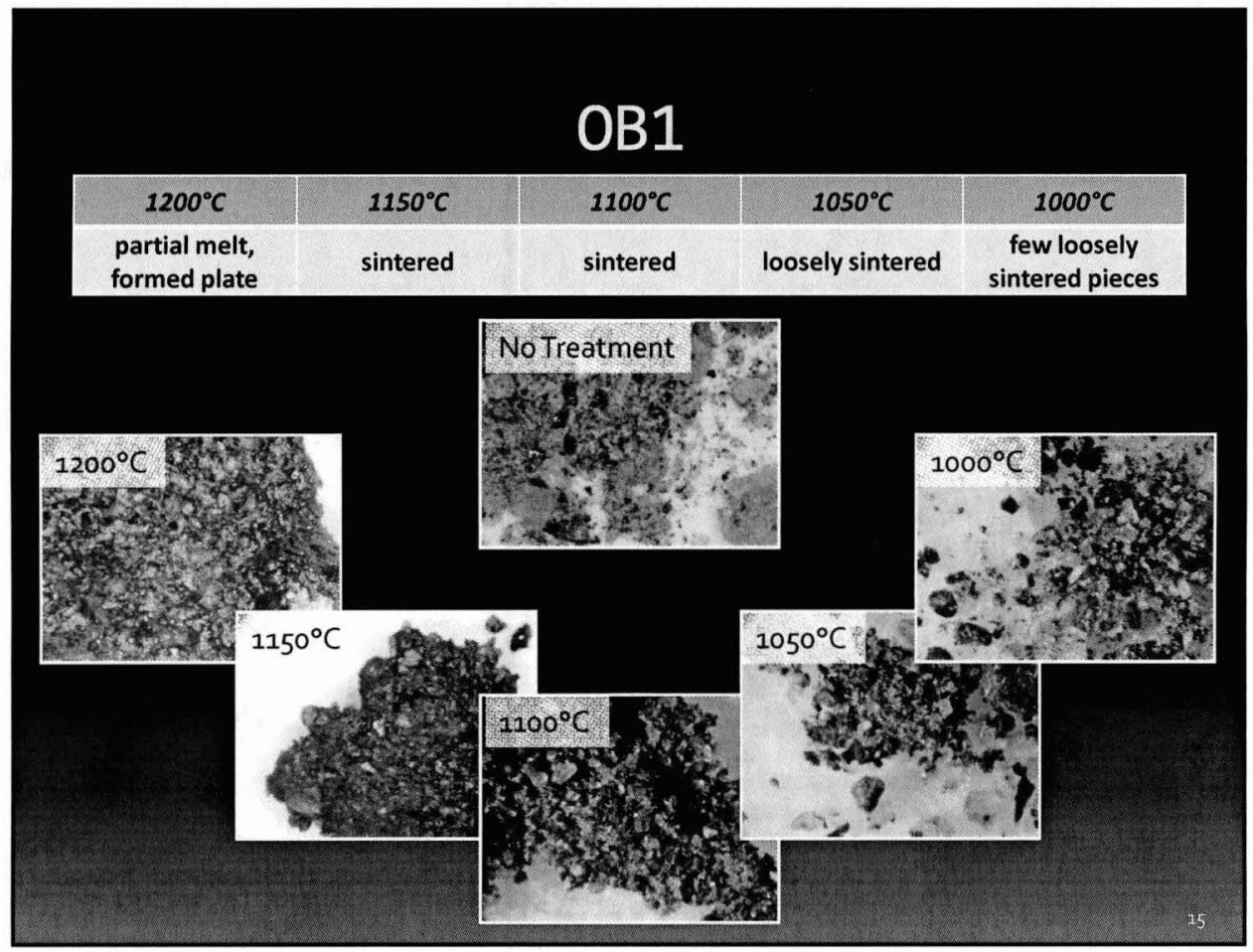

\section{Simulant Laboratory Study:}

OB-1

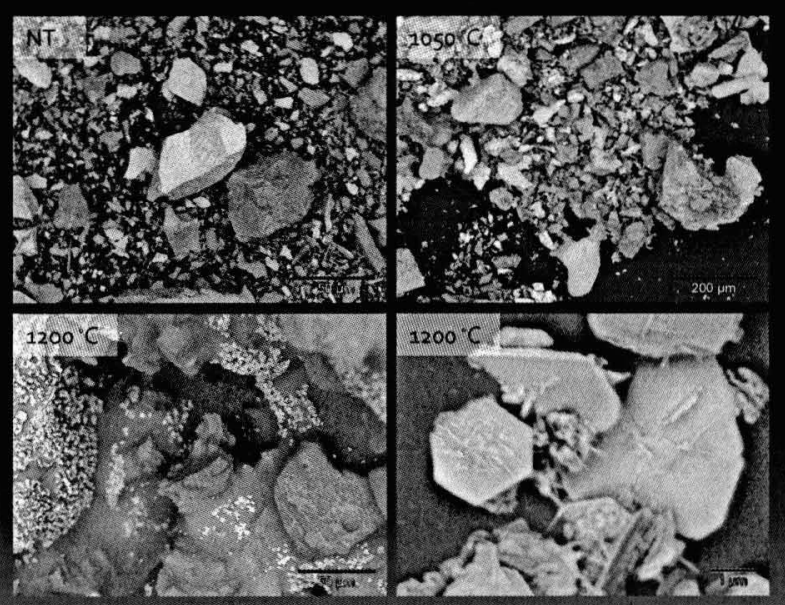




\section{Simulant Laboratory Study:}

\section{Conclusions}

- Sintering occurs when on phase melts or starts to flow onto other particles

- Glasses melt and flow at lower temperatures

- Calcium rich plagioclase was the last mineral to melt in all simulants

- OB-1 and NU-LHT-2m behaved similarly, due to glass phase and high melting point phase

- Simulants for sintering should have a high melting point mineral and glass phase to match desired regolith composition (for lunar case)

\section{Field Demonstrations}

- Hawaii field demo

- Masten Space Systems

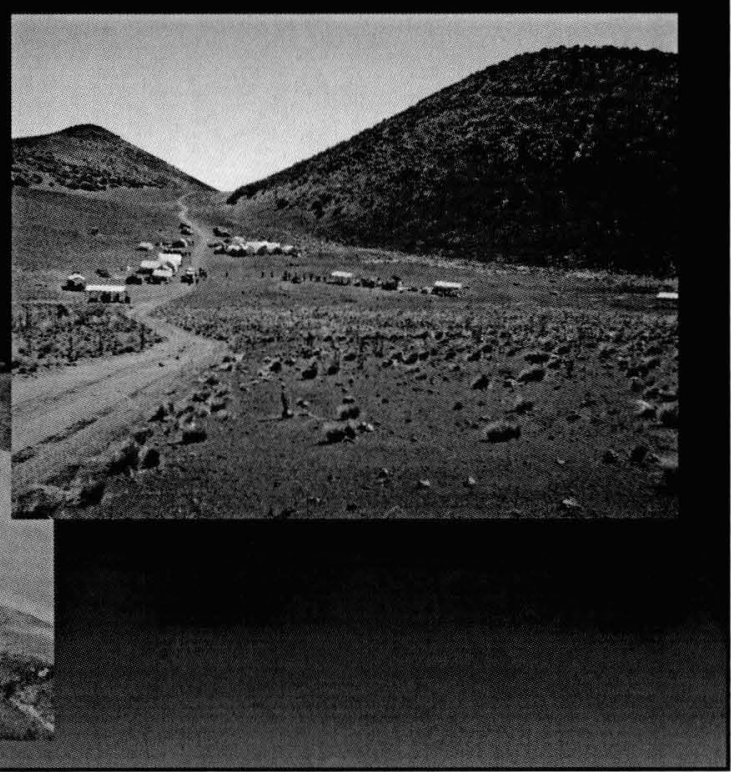




\section{Mauna Kea, Feb. 2010}

- Large Area Surface Sintering System (LASSS)

- Uses resistive heater

- Incorporates layered sintering and temperature feedback

- Mounted on NORCAT rover
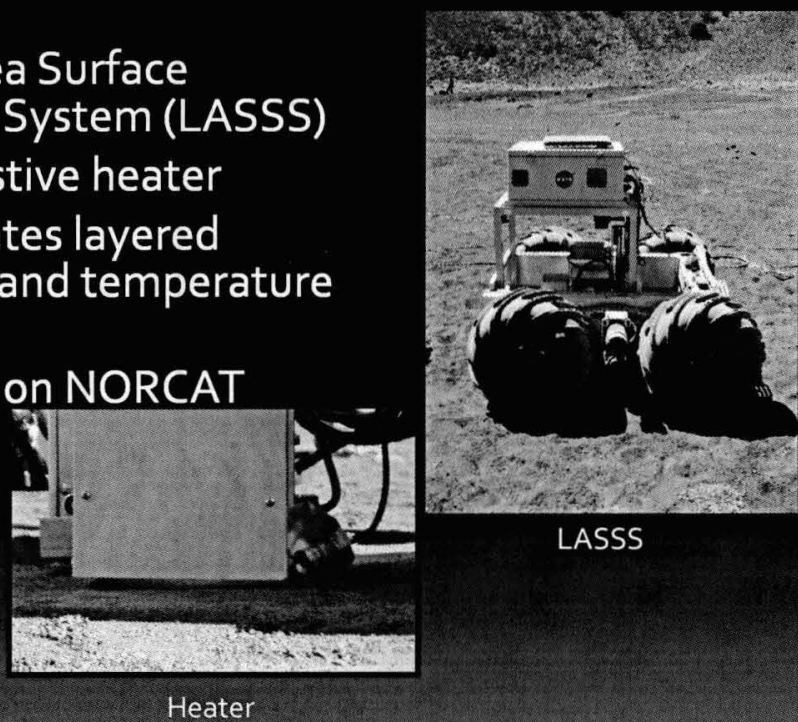

\section{Mauna Kea, Feb. 2010}

- Able to layer tephra and connect sintered areas

- Strengths from $30-240$ psi

- Fired thruster on sintered area

- Environmental conditions caused issues
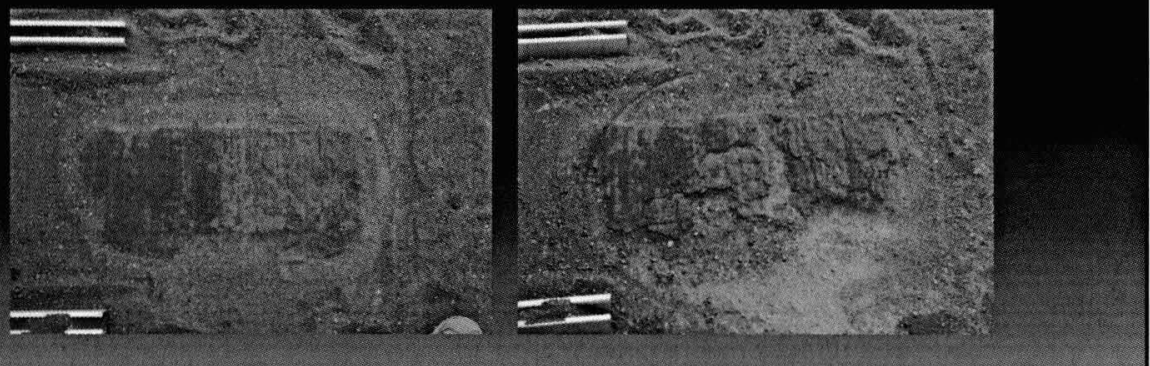


\section{Masten Space Systems: Nov 2011}

- Sample coupons were mounted on a steel plate and placed near the vehicle exhaust

- Fondu Fyre (used in LC39 flame trench)

- Sintered JSC-1A tiles

- Polymer/Regolith Composites (Adherent Technologies)

- Slicone Ablative (LC39)

- Kevlar and Carbon fiber

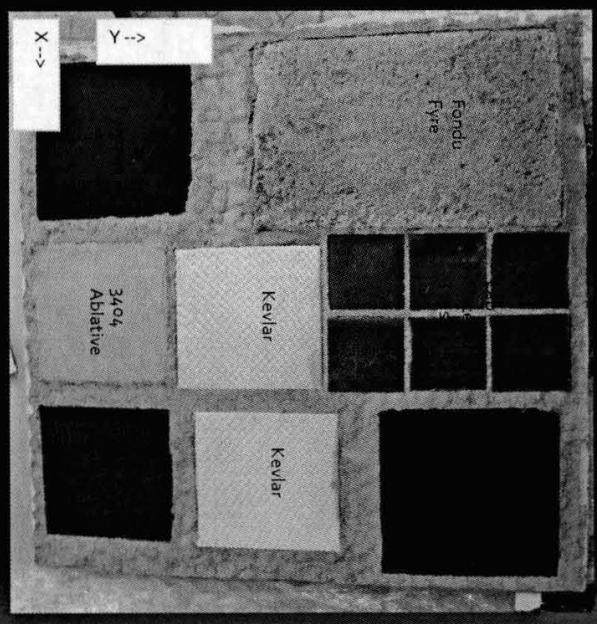

\section{Masten Space Systems: Nov 2011}
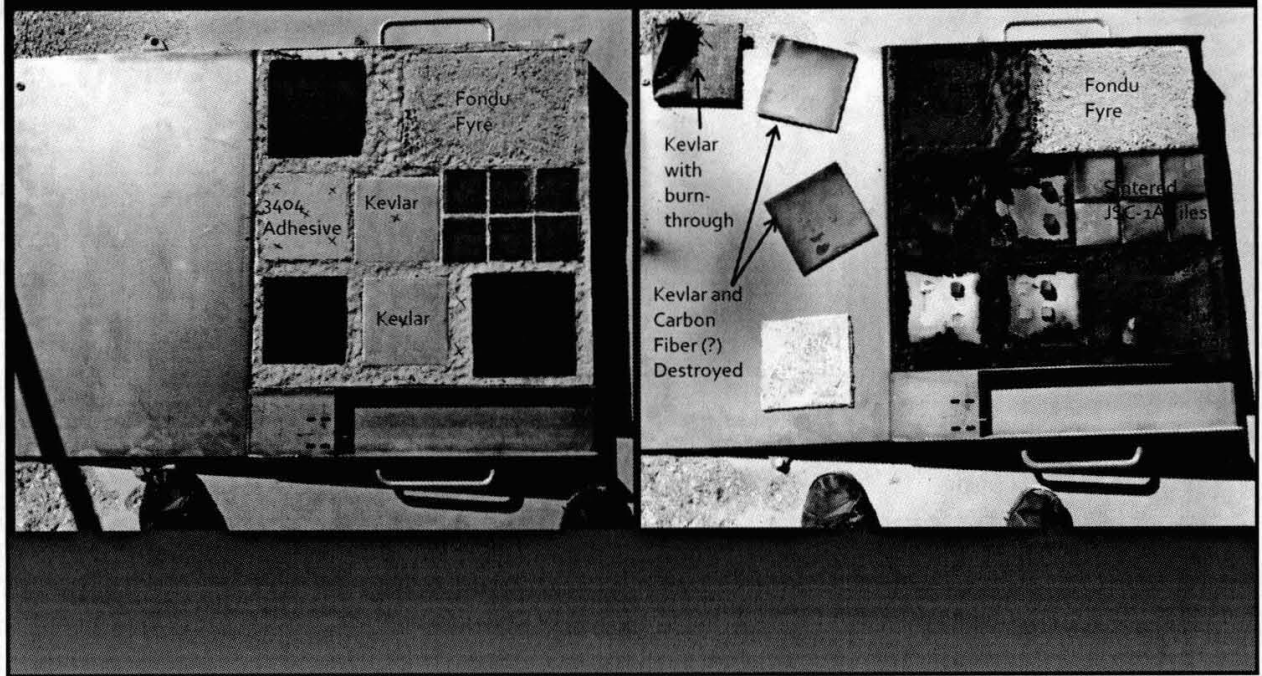


\section{Masten Space Systems: Nov 2011}

- Sintered Regolith - Little to no erosion

- Polymer/regolith composite

- Did well considering it was not designed for this

- LC39 materials - Expected performance

- Fabrics failed due to poor attachment to plate
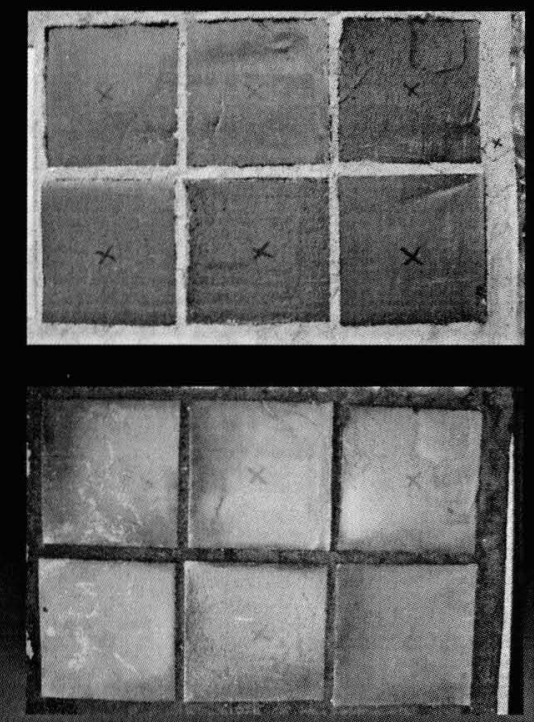

\section{Acknowledgements}

- NASA KSC

- Phil Metzger

- Rob Mueller

- Janine Captain

- David Smith

- Luke Roberson

- ASRC

- Jerry Curran

- Teddy Back
- Chris Immer

- Mike Csonka

- Interns

- Brittany Griffin 